\title{
Les postfigurations du loisir. Vers une typologie des réflexions autobiographiques
}

The Postfigurations of Leisure. Towards a Typology of Autobiographical Reflexions

\section{Georg Feitscher}

Traducteur : Cécile Roche

\section{OpenEdition}

\section{Journals}

Édition électronique

URL : https://journals.openedition.org/recherchestravaux/826

DOI : $10.4000 /$ recherchestravaux.826

ISSN : 1969-6434

\section{Éditeur}

UGA Éditions/Université Grenoble Alpes

\section{Édition imprimée}

Date de publication : 1 juin 2016

Pagination : 157-176

ISBN : 978-2-84310-325-4

ISSN : 0151-1874

\section{Référence électronique}

Georg Feitscher, "Les postfigurations du loisir. Vers une typologie des réflexions autobiographiques », Recherches \& Travaux [En ligne], 88 | 2016, mis en ligne le 01 janvier 2017, consulté le 29 octobre 2021. URL : http://journals.openedition.org/recherchestravaux/826 ; DOI : https://doi.org/10.4000/ recherchestravaux.826 


\section{Les postfigurations du loisir. Vers une typologie des réflexions autobiographiques}

(Traduit de l'allemand par Cécile Roche)

\section{Loisir et autobiographie}

Les réflexions théoriques sur le loisir se sont toujours efforcées de différencier celui-ci de tout mode d'action fonctionnel. Dans les textes programmatiques et scientifiques, il est expliqué que le loisir offre la possibilité d'une "prise de distance avec les tracas quotidiens", en particulier des activités salariées ou commerciales que l'on a l'habitude d'exercer (negotium ${ }^{\mathrm{r}}$ ). À l'inverse de ces occupations, les pratiques de loisir constituent «un plaisir pur libéré de toute finalité ${ }^{2}$. Elles sont caractérisées par l'absence de finalité en dehors d'ellesmêmes : "le loisir n'est pas un agir rationnel et fonctionnel"», il ne peut en aucun cas "être tendu vers une finalité dirigée vers le futur ${ }^{4}$ ».

On retrouve l'origine de ces descriptions contemporaines du loisir jusque chez Aristote. Il différencie les activités exercées "dans le cadre de la vie civique» comme les fonctions étatiques et le service militaire de la theoria, qui «ne tend

I. C. Wulf et J. Zirfas, «Die Muße. Vergessene Zusammenhänge einer idealen Lebensform », dans C. Wulf et J. Zirfas (dir.), Muße, Berlin, Akademie-Verlag, 2007, p. 9.

2. F. Hessel, «Von der schwierigen Kunst spazieren zu gehen» [1932], Die Horen, n 4, 2000, p. 46.

3. C. Wulf et J. Zirfas, «Die Muße», art. cité, p. II.

4. H. Brühweiler, Musse (scholé). Ein Beitrag zur Klärung eines ursprünglich pädagogischen Begriffs, Zurich, Benziger, I97I, p. Io. 
à aucune fin en dehors d'elle-mêmes". Pour Aristote, la pure contemplation spirituelle de soi de la theoria est une condition essentielle à la conduite d'une vie heureuse (eudaimonia) : "car le bonheur n'a besoin de rien d'autre que lui et se suffit à lui-même ${ }^{6} »$.

Suivant cette tradition, le concept de loisir (et ses équivalents dans d'autres langues) n'a cessé d'être employé dans l'histoire pour désigner cette posture et ce mode de vie qui s'écartent du mode fonctionnel dominant de la société, c'est-à-dire de toutes les activités quotidiennes et immédiatement nécessaires dont on doit s'acquitter pour assurer la survie et la pérennisation de la société. Pourtant, c'est précisément parce que le loisir n'entretient pas un rapport de cause à effet avec un mode fonctionnel primaire (aujourd'hui sans doute pleinement économique), qu'il s'ouvre à des fonctionnalités d'un autre genre, qui se sont révélées extrêmement changeantes au cours de l'histoire.

Ainsi, l'otium était au Moyen Âge un élément important dans une praxis religieuse, qui se focalisait sur la reconnaissance de la puissance de Dieu et la participation à son règne. La contemplation du moine ou même de l'ermite qui prend Dieu pour objet et, plus tard, les expériences spirituelles des mystiques ont construit le paradigme d'un loisir doté d'une fonction religieuse. Le loisir a été élevé au rang d'un mode de réflexion, qui n'est pas au service du particulier, mais de l'État ou de la communauté religieuse ${ }^{7}$.

Avec le passage dans la modernité s'est construite une conception moderne de l'individualité humaine. Parallèlement, le potentiel réflexif attaché au loisir est de plus en plus dirigé vers le sujet qui se découvre en lui. À partir de là, le loisir est considéré comme un espace de liberté privilégié, qui se passe désormais de légitimation sociale ou religieuse, mais qui s'ouvre au contraire à une expérimentation entièrement subjective. Au lieu de contempler Dieu et l'ordre divin, l'individu moderne se place lui-même au centre de sa perception, ou comme le dit Rousseau : «De quoi jouit-on dans une pareille situation? De rien d'extérieur à soi, de rien sinon de soi-même et de sa propre existence ${ }^{8}$ ». Le loisir en vient à désigner une situation qui sort de l'ordinaire, dans laquelle

5. Aristote, L'Éthique à Nicomaque, trad. R. A. Gauthier et J. Y. Jolif, Louvain, Peeters, 1972, p. 308 (X, 7, II77b).

6. Ibid., p. $30 \mathrm{OI}$ (X, 6, 1176b).

7. Saint Augustin donne une définition normative de cet idéal : "In otio non iners vacatio delectare debet, sed aut inquisitio aut inventio veritatis, ut in ea quisque proficiat et quod invenerit ne alteri invideat." - "Dans l'inaction [otium], on ne doit pas aimer le repos [vacatio] pour lui-même, mais s'adonner à la recherche de la vérité afin d'en bénéficier soi-même et de ne pas en jalouser autrui.» (A. Augustinus, Der Gottesstaat / De civitate Dei. In deutscher Sprache von C. J. Perl, vol. 2, Paderborn, Schöningh, 1979 [413-426 P.C.N.], XIX, I9, p. 490.)

8. J.-J. Rousseau, Les Rêveries du promeneur solitaire, dans CEuvres complètes, éd. B. Gagnebin et M. Raymond, vol. I, Paris, Gallimard, I959, p. 993-1097, p. I047. (Désormais OC, I.) 
l'individu pratique l'introspection, «le temps où l'on est auprès de soi" ». Par conséquent, comme l'explique Schopenhauer au milieu du XIX ${ }^{\mathrm{e}}$ siècle, «les loisirs acquis par chacun, en lui donnant la libre jouissance de sa conscience et de son individualité, sont à ce titre le fruit et le revenu de toute son existence ${ }^{\mathrm{IO}}$ ».

Les remarques de Schopenhauer dans les Aphorismes sur la sagesse de la vie témoignent de la réorientation moderne du loisir sur le sujet. La constitution autonome de l'individu devient la fonction la plus importante du loisir moderne; et la narration devient sa principale technique. À travers le médium de la narration, l'être humain peut donner du sens à sa vie et se construire luimême une identité narrative $e^{\text {II }}$. Cette idée se manifeste principalement dans le genre littéraire de l'autobiographie, qui rencontre un immense succès depuis la fin du XVIII ${ }^{\mathrm{e}}$ siècle. L'autobiographe classique se distingue en ce qu'il intègre les expériences hétérogènes qu'il a vécues dans sa vie dans une narration totalisante et les ordonne le long d'un fil narratif. Dans leurs autobiographies canoniques, Rousseau et Goethe en particulier ont très profondément marqué les représentations du narrateur autobiographe, lequel rétrospectivement, dispose librement de ses propres souvenirs et les interprète afin de se construire une identité personnelle solide.

En conséquence, la situation dans laquelle se trouve le narrateur autobiographe est elle-même décisive pour la réussite de cette rétrospective. Ce n'est que dans un cadre bien particulier que la mise en narration de sa propre vie apparaît comme possible, légitime et prometteuse. Il n'est guère étonnant de voir que les autobiographes classiques dépeignent souvent ce cadre comme une situation de loisir. Le loisir est élevé au rang de condition préalable à la réflexion et à la narration autobiographiques et donc, à la réussite de la constitution autonome de l'individu. Bien plus, on peut affirmer que le discours moderne sur le loisir a pour principale fonction de donner la clé du concept de sujet. Le paradigme individualiste sous-entend en effet qu'il y aurait un lieu ou un temps, qui permettraient à l'individu d'être en accord total avec lui-même et de favoriser la réalisation de soi. Le concept de loisir sert aujourd'hui à désigner une structure spatiotemporelle utopique, qui est, dans une certaine mesure devenue, indispensable aux conceptions modernes d'individualité autonome et d'aspiration à la réalisation de soi.

9. M. Renouard, "L'otium entre politique et rêverie", dans M. Fumaroli, D. Simon, J.-C. Darmon et G. Métayer (dir.), L'otium dans la République des lettres, Paris, CNRS, A. Baudry, 20II, p. 74 .

Io. A. Schopenhauer, Aphorismes sur la sagesse de la vie [1851], éd. G. Heff, trad. de l'allemand J. A. Cantacuzène, 20I3, p. 32 (http://www.schopenhauer.fr/oeuvres/fichier/aphorismes.pdf). II. Voir P. Ricour, «L'identité narrative», Esprit, n 7-I2, I988, p. 295-304. 
Le lien entre loisir, narration et réussite de la constitution da sa propre identité est structuré à l'aide de topoï par de nombreux auteurs. La littérature autobiographique déploie un large répertoire de lieux et de pratiques, qui permettent au narrateur de se mettre en situation de loisir. Ils se présentent comme des modèles que l'on a repris jusque dans les récits autobiographiques des quarante dernières années. Je tenterai de regrouper sous le concept de postfigurations ces approches contemporaines de la constitution autonome du sujet par le loisir, dans lesquelles il s'agit de reprendre des topö̈ établis et conventionnels. Je me concentrerai donc sur deux textes qui laissent entrevoir ces représentations postmodernes du loisir présentées de façon positive. Afin de mettre en évidence les recours à des topoï plus anciens, il faut s'appliquer à comparer ces textes avec un modèle classique qui les préfigure. Comme elle ne constitue qu'un extrait restreint du spectre de la narration autobiographique contemporaine, cette présentation ne prétend bien sûr pas à l'exhaustivité, mais elle n'est cependant pas tout à fait arbitraire. Les personnages, lieux et pratiques dont il sera ici question sont de ceux qui ont durablement marqué l'histoire de l'introspection littéraire.

\section{Promenades}

Avant Rousseau, la nature et le paysage n'étaient que très rarement l'objet de considérations esthétiques; bien plus, ils étaient «méprisés " par les voyageurs parce qu'ils étaient considérés comme «une perte de temps, un espace intermédiaire séparant les lieux dont l'on peut tirer profit ${ }^{12}$ ». La narration autobiographique semble toutefois faire exception. Mikhaïl Bakhtine attire l'attention sur le fait que la déconverte du paysage qui conduit à une mise en relation très étroite de la nature extérieure avec la subjectivité intérieure influençait déjà les (auto)biographes de l'Antiquité :

Alors naît le "paysage», c'est-à-dire la nature comme horizon (objet de vision) et environnement (fond, décor) de l'homme privé, solitaire et passif. [...] Dans le monde clos de l'homme privé, la nature pénètre par fragments pittoresques, aux heures de promenade, de repos, lorsqu'on jette un regard fortuit sur un paysage qui se découvre ${ }^{13}$.

12. P. Raymond, Von der Landschaft im Kopf zur Landschaft aus Sprache. Die Romantisierung der Alpen in den Reiseschilderungen und die Literarisierung des Gebirges in der Erzählprosa der Goethezeit, Berlin, de Gruyter, I993, p. 6r.

I3. M. Bakhtine, Esthétique et théorie du roman, trad. du russe D. Olivier, Paris, Gallimard, p. 290. 
La promenade, qui constitue un mouvement en apparence sans but, est une forme de rencontre entre l'homme et le paysage spécifique de la modernité. Dès la fin du XVIII ${ }^{\mathrm{e}}$ siècle, la promenade correspond à une pratique de loisir très populaire et accessible au plus grand nombre, qui offre à la plupart des sujets modernes la possibilité non seulement de contempler la nature en dehors des grandes villes à la croissance spectaculaire, mais aussi de pratiquer l'introspection. Ainsi «l'attention particulière accordée de nos jours au promeneur oisif $^{14}{ }^{\star}$ n'est que l'actualisation la plus récente d'un topos du loisir en réalité très ancien. Les motivations intrinsèques au loisir et à la promenade sont les mêmes, et de la même façon, la définition de la promenade peut s'appliquer à l'oisiveté : «Elle n'a en apparence aucune finalité, elle reste dans une sphère limitée, [...]. Elle n'est pas un moyen pour atteindre un but, elle est ce but ellemême ${ }^{15}$. On peut sans doute expliquer dans ce sens la fréquence des voyages et des promenades dans la littérature contemporaine : par «l'aspiration à se soustraire à ses devoirs ${ }^{16}$ ", et donc par l'aspiration au loisir. La littérature a la particularité de réactualiser sans cesse la figure du promeneur oisif, parce qu'il y a peu d'autres pratiques qui entretiennent un lien étroit avec la littérature comme le fait la promenade, qui occupe de ce fait une place prépondérante dans les entreprises introspectives et poétologiques des auteurs. Le principe de cette pratique repose sur une "stylisation imaginaire de la promenade, où les chemins de terre deviennent des pages de carnet, et où l'on écrit, non pas en marchant - ce qui est peu pratique - mais après avoir marché, la dynamique des pensées relayant celle des pas en avant ${ }^{17}{ }^{17}$.

Cette observation de Jacques Lecarme et Éliane Lecarme-Tabone a été spécialement conçue pour les écrits autobiographiques de Rousseau. À Rousseau revient le mérite d'avoir mis en place ce topos qui lie le loisir de la promenade à l'écriture introspective, qui a fait forte impression sur la littérature européenne. Contrairement aux flâneurs urbains, plus tardifs, qui n’apparaissent pas seulement comme les observateurs de la culture de masse urbaine, mais qui s'exposent eux aussi au regard des gens, Rousseau met en place l'image d'un promeneur solitaire qui tourne son regard en direction de sa propre intériorité : «Jamais je n’ai tant pensé, tant existé, tant vécu, tant été moi, si j’ose

I4. F. Schäfer, «Ich bleib dann mal hier. Die Wiederkehr des Flaneurs», Rolling Stone, $\mathrm{n}^{\circ}$, 20I2, p. 57.

I5. A. Montandon, Sociopoétique de la promenade, Clermont-Ferrand, Presses universitaires Blaise Pascal, 2000, p. 7.

I6. F. Schäfer, «Ich bleib dann mal hier...», art. cité, p. 57.

I7. J. Lecarme, É. Lecarme-Tabone, L’Autobiographie, Paris, Colin, 1997, p. I55. F. Schäfer, "Ich bleib dann mal hier...", art., cité, p. 57. 
ainsi dire, que dans [les voyages] que j'ai faits seul et à pied.» $\left(O C, I, \mathrm{p} \cdot 162^{18}\right)$ Avec cette constatation du livre IV de ses Confessions, Rousseau souligne la signification essentielle que revêt la marche pour la constitution de sa propre identité. Ailleurs, il reconnaît la promenade comme une praxis, qui décuple sa productivité littéraire : «Je destinai, comme j'avois toujours fait [...] mes après-dînées à la promenade, muni de mon petit livret blanc et de mon crayon [...] et je comptois bien que la forest de Montmorency qui étoit presque à ma porte, seroit désormais mon cabinet de travail». (OC, I, p. 404)

Dans les Rêveries du promeneur solitaire, un nouveau lieu de retraite s'offre à Rousseau et remplace la forêt. Dans un commentaire célèbre, Rousseau explique dans la première promenade qu'il poursuit à travers l' « informe journal de mes rêveries» (OC, I, p. IOoo) deux buts différents : «Il y sera beaucoup question de moi parce qu'un solitaire qui réfléchit s'occupe nécessairement beaucoup de lui-même» (ibid.). À l'aide de son journal, Rousseau veut en premier lieu se créer un nouvel espace textuel qui lui permette de pratiquer l'introspection et ce faisant, parvenir à « une nouvelle connoissance de [son] naturel et de [son] humeur» (ibid.). En second lieu, il fait de la promenade une praxis intellectuellement créative et productive : «Du reste toutes les idées étrangéres qui me passent par la tête en me promenant y trouveront egalement leur place.» (Ibid.)

Introspection et promenade sont très étroitement liées l'une à l'autre dans les carnets de Rousseau. Il trouve cependant dans le loisir de ses promenades sur l'île Saint-Pierre non seulement un motif thématique, mais aussi la métaphore d'un processus narratif. La division de son œuvre en dix promenades constitue la tentative la plus évidente de donner à ses réflexions une structure textuelle qui soit à leur mesure. Les raisonnements fragmentaires de Rousseau, qui pense par associations d'idées, ne correspondent plus seulement à un principe épistémologique, mais sont élevés par lui au rang de forme littéraire.

Dans la "Cinquième promenade», Rousseau explique en détail pourquoi l'île est un lieu de retraite particulièrement adapté aux promenades :

Mais il faut avouer que cela se faisoit bien mieux et plus agréablement dans une Isle fertile et solitaire, naturellement circonscritte et séparée du reste du monde, [...] où je pouvois enfin me livrer tout le jour sans obstacle et sans soins aux occupations de mon gout, ou à la plus molle oisiveté» (OC, I, p. I048).

L'île constitue un lieu prédestiné au loisir et à l'introspection pour une autre raison :

18. Voir aussi J. Plessen, Promenade et poésie. L'expérience de la marche et du mouvement dans l'œuvre de Rimbaud, Paris, Mouton et Cie, 1967, p. 67-70. 
Quand le soir approchoit [...] j'allois volontiers m'asseoir au bord du lac sur la gréve dans quelque azyle caché; là le bruit des vagues et l'agitation de l'eau fixant mes sens et chassant de mon ame toute autre agitation la plongeoient dans une réverie delicieuse où la nuit me surprenoit souvent sans que je m'en fusse apperceu. Le flux et reflux de cette eau, son bruit continu mais renflé par intervalles frappant sans relache mon oreille et mes yeux suppléoient aux mouvemens internes que la rêverie éteignoit en moi et suffisoient pour me faire sentir avec plaisir mon existence, sans prendre la peine de penser. (OC, I, p. I045)

Cernée de toutes parts par le lac, la rive offre au narrateur la possibilité de se regarder dans l'eau et de s'y réfléchir à loisir : «[...] laissant errer mes yeux au loin sur les romanesques rivages qui bordoient une vaste étendue d'eau claire et cristalline, $[. .$.$] et me trouvant enfin ramené par degrés à moi-même et à$ ce qui m’entouroit [...]» $(O C, I$, p. I048).

De nombreux exemples montrent que les promenades de Rousseau ont donné une représentation du loisir qui est, encore jusqu'à nos jours, profondément ancrée dans notre environnement culturel. Les auteurs font encore référence à cet état d'esprit des générations après. Dans, Montauk ${ }^{19}$, écrit d'inspiration autobiographique de Max Frisch paru en I975, les promenades au bord de l'eau jouent un rôle décisif dans le projet de remémoration de l'auteur. L'élément déclencheur de cette réflexion est un voyage entrepris par Frisch à travers les États-Unis pour la promotion d'un livre, dont la dernière étape le conduit à New York. C'est là que Frisch, alors âgé de 63 ans, entreprend une liaison avec Lynn, employée d'une maison d'édition, dont il est de plusieurs dizaines d'années l'aîné. Dans ce récit rédigé sous forme de fragment, le narrateur se présente comme un flâneur dans New York, mais avant tout comme un promeneur qui marche le long de la presqu'île située à l'est, Long Island. Il y entreprend en effet un voyage en compagnie de sa jeune compagne durant le week-end du in au i2 mai i974.

Le séjour à New York est déjà décrit par le narrateur comme une période d'oisiveté. Max Frisch abandonne, temporairement du moins, son rôle d'artiste et d'intellectuel : au lieu de regarder la nouvelle exposition du musée d'Art moderne, il se contente de rester assis "dans le jardin, toute une matinée» $(M O$, p. I5). Au cours de ses flâneries à travers la ville, il découvre « [q] uelques personnes sur le môle, des oisifs, comme [lui]. [...] Un vieux avec un chien. Un autre chien sans maître.» (MO, p. I5) Le regard indifférent de Frisch rencontre des personnes ou des objets, les juxtapose sans logique apparente. Les objets contemplés entretiennent toutefois une relation symbolique avec le narrateur : ils sont âgés comme lui, solitaires comme lui. Même la description d'une «boîte

I9. M. Frisch, Montauk, trad. M. et J. Tailleur, Paris, Gallimard, 1978. (Désormais MO.) 
de bière qui se met à rouler dans le vent» (MO, p. I5) peut être lue comme un commentaire introspectif sur le narrateur, qui, de la même façon, se laissera bientôt porter par le destin dans les dunes de Long Island.

L'excursion dominicale, marquée par une douceur mélancolique, est en totale rupture avec le quotidien de l'auteur. En voiture, Max et Lynn suivent un panneau indiquant "OVERLOOK», qui leur promet "un point de vue» intéressant sur l'île. Ils doivent toutefois abandonner rapidement la voiture pour se frayer un chemin à pied à travers la nature : "buissons et fourrés autour du parking vide; pas de point de vue donc, mais un sentier traverse les fourrés, et ils n'ont pas discuté longtemps : le sentier va les conduire au point de vue grandiose» $(M O$, p. 9). De toute évidence Max n'est pas seulement à la recherche d'un joli point de vue qui lui offrirait une vue dégagée sur le paysage. Bien plus, il trouve dans Long Island un cadre qui lui offre la possibilité de contempler le paysage et de revenir sur sa vie, et qui l'y contraindrait même peut-être dans la mesure où sa liaison avec la jeune Lynn lui fait sentir le poids des ans et tirer un bilan. C'est pourquoi le récit du week-end à Montauk est constamment entrecoupé de phases plus ou moins courtes de remémoration de sa vie. Il en résulte une structure faite de récits encadrants et encadrés : le narrateur décrit ses aventures à Long Island au présent, mais parle souvent de lui, si ce n'est constamment, de façon distanciée, à la troisième personne, comme s'il s'observait de l'extérieur.

Entre ces descriptions, il convoque le souvenir des événements significatifs de sa vie, pour la plupart racontés à la première personne du passé, selon la grammaire classique de l'autobiographie ${ }^{20}$. Le récit encadrant, le week-end à Long Island, éclaire les réflexions sur la thématique du récit autobiographique qui sont abordées ici. En effet, les représentations véhiculées par Frisch mettent très nettement en avant le cadre spatiotemporel et les conditions pratiques auxquelles est liée sa réflexion.

C'est par «une longue après-midi légère», que Max et Lynn atteignent l'extrémité la plus à l'est de Long Island :

Une plage longue de plusieurs miles, de la plage à perte de vue, qui se perd des deux côtés dans le lilas laiteux de l'évaporation. Malgré le vent, la chaleur est presque torride. Deux transats aux coussins passés sont installés dans le sable, sans aucun autre à la ronde; à qui appartiennent-ils? Pas d'âme qui vive à la ronde. (MO, p. 79)

20. À l'inverse, A. Krättli observe un emploi tout à fait «irrégulier » des pronoms personnels qui expriment tout au plus « différents degrés d'implication dans le rôle de l'écrivain ", "Leben im Zitat". Max Frisch Montauk», dans W. Schmitz (dir.), Über Max Frisch, Francfort-sur-leMain, Suhrkamp, 1976, p. 43I-432. 
Ce n'est qu'à une centaine de miles de l'activité de la ville monde que Max et Lynn se retrouvent dans une complète solitude. Ils répondent avec plaisir à l'invitation au repos de ces deux chaises longues rencontrées par hasard sur la plage ${ }^{21}$. Au matin du jour suivant, le narrateur se promène seul en face de l'Atlantique («Mieux vaut enlever ses chaussures et marcher pieds nus dans le sable, les chaussures à la main.») et décrit le paysage solitaire qui l'entoure : «la mer gris perle le matin sous les nuages bas, le ressac sans force, pas de soleil» $(M O, \mathrm{p}$. II2). C'est là que s'ouvre alors au narrateur l'horizon tant recherché. Il n'a qu'une envie : "qu'ils regardent maintenant au loin [...] pour que, ce faisant, il s'oublie : un homme trop lourd [...] les cheveux gris-blanc...» (MO, p. 47). L'immensité de l'océan permet à Frisch de porter son attention sur l'eau, loin de lui-même, en espérant pouvoir, au moins pour un temps, oublier son propre présent d'homme avancé dans l'âge. Mais l'instant d'après, l'attention du narrateur est déjà reportée sur lui-même, ce n'est plus sur ses dispositions présentes qu'il se concentre mais sur son passé : «Il lui vient à l'esprit quand il s'est baigné pour la dernière fois dans la mer.» (MO, p. 47)

Considérée à part, cette citation donne presque l'impression que le narrateur use d'une conception nostalgique du récit autobiographique dans laquelle le regard rétrospectif sur ce qui a été accompli offrirait en quelque sorte la possibilité de fuir un présent décevant. Ses impressions sont toutefois plus ambivalentes. Certes, le repos présent est douloureux pour le narrateur, parce que cela lui fait prendre conscience de son âge, mais il prend aussi plaisir à la rupture avec la routine quotidienne et à la possibilité de réfléchir sur sa vie qui se présente lors de ses pérégrinations sur Long Island. En conséquence, le narrateur souligne à maintes reprises sa capacité de s'investir dans le présent et s'essaie à différentes pratiques, afin de se forger une expérience du repos présent, comme le fait de bourrer sa pipe :

Il a tapoté sa pipe pour la vider et pense : [...] bourre la pipe avec le pouce droit et cette précaution qui occupe les instants, des instants sans mémoire [...] et avec précaution tire la première bouffée, courte, puis une deuxième, longue, jusqu’à ce que tu sois tout à fait là. Pour l'instant, il n'y a qu'eux deux dans les transats [...]. $(M O$, p. $8 \mathrm{I})$

Le narrateur invoque l'instant présent comme un leitmotiv qui le libère de toute finalité orientée vers le futur : «le monde se dérobe dans son avenir sans moi, et de là ce repliement sur le moi qui se sait exclu de la communauté d'avenir " (MO, p. II4). L'expérience du présent se fait plus intense pour le narrateur paré

2I. À propos du raport entre repos et réalisation de soi, voir M. Theunissen, Negative Theologie der Zeit, Francfort-sur-le-Main, Suhrkamp, I99I, p. 29I-292. 
contre le passé et le futur ${ }^{22}$. Dans son expérience subjective, le présent est étiré sur une immense durée et devient "vide absolu : quand un quart d'heure, le prochain quart d'heure, paraît plus long que l'année écoulée» ( $M O$, p. II5).

L'expérience du présent se révèle toutefois rapidement fragile, et le découplage du temps et du quotidien que permet le loisir éphémère : "[le narrateur] n'oublie pas son rôle, ni les prochaines obligations qui découlent de ce rôle; des dates à respecter, il n'oublie même pas la situation mondiale. Toute une foule de choses qu'il n'oublie pas dans ce présent ténu.» $(M O$, p. 85-86) À cet endroit, Frisch met l'accent, même si ce n'est pas la première fois, sur l'image qu'il se fait de lui-même en tant qu'auteur engagé politiquement. Il se considère trop lié au « rôle» de l'observateur et commentateur de la société pour pouvoir s'en dégager si facilement. En fait, les réflexions idiosyncratiques telles que Frisch les conduit dans Montauk ne correspondent pas à sa conception de la littérature : «[1]a littérature a un autre temps, en outre un sujet qui concerne tout le monde ou beaucoup - ce qu'on ne peut dire des deux chaussures qu' elle a laissées dans le sable...» $(M O$, p. 85). Et c'est précisément la littérature qui arrache Frisch au présent ténu de l'instant et menace de porter sa réflexion à un niveau supra-individuel : «[1]a littérature conserve l'instant, c'est pour cela qu'elle existe» $(M O$, p. 85$)$.

Cette confession sur la littérature politique, glissée à cet endroit presque comme une citation autoréférentielle ironique, est en contradiction pragmatique avec le récit Montauk lui-même, qui compte sans conteste parmi les œuvres introspectives les plus personnelles et ostentatoires de Frisch. En effet, on peut considérer Montauk comme un tournant poétologique au sein des œuvres de vieillesse de Frisch, un tournant dont il débat dans le récit lui-même. Si Frisch a jadis décrit la Sphère publique comme partenaire de l'auteur ${ }^{23}$, il voit à présent la relation entre écrivain et société sous un jour problématique. Il se plaint d'avoir «approvisionné je ne sais quelle opinion publique en histoires. Dans ces histoires, je me suis mis à nu, je le sais, à en être méconnaissable». Certes, son œuvre était fondée sur son propre vécu, mais il n’y avait que les "parties, dont [il a] pu faire de la littérature» (MO, p. I27) qui l'intéressaient.

À cette trahison de lui-même, le narrateur oppose une méditation sur l'intériorité et l'introspection. Il explique qu'il voudrait «ne rien inventer» : il

22. Sur l'expérience du présent et de la modernité chez Frisch voir aussi R. Hartung, " "Schreibend unter Kunstzwang". Zu der autobiographischen Erzählung Montauk von Max Frisch", dans W. Schmitz (dir.), Über Max Frisch, Francfort-sur-le-Main, Suhrkamp, 1976, p. 438-439.

23. Thème du discours tenu par Frisch pour l'ouverture de la Foire aux livres de Francfort, publié sous le titre «Öffentlichkeit als Partner», dans Öffentlichkeit als Partner, Francfort-surle-Main, Suhrkamp, 1967, p. 56-67. 
s'agit seulement de «savoir ce que je perçois et pense lorsque je ne pense pas à des lecteurs possibles. Est-ce que j'écris donc pour satisfaire des lecteurs, pour approvisionner des critiques!» ( $M O$, p. II3) Le récit du week-end à Montauk offre à Frisch une occasion bienvenue pour l'introspection, mais aussi pour l'exploration d'une écriture subjective nouvelle : le narrateur se propose de donner au récit une forme "autobiographique, oui, autobiographique» ( $M O$, p. 126). Ce tournant vers l'intériorité présente un nouveau programme narratif, esquissé par Frisch dans l'une de ses réflexions : «je voudrais décrire cette journée, rien que cette journée, notre week-end, et comment cela est arrivé, comment cela se poursuit. Je voudrais pouvoir raconter sans rien inventer. Une position simpliste de narrateur» $(M O$, p. 69).

Frisch peut s'attaquer sans attendre aux défis qu'il découvre alors dans l'écriture pour soi, car toujours à New York, il s'est procuré le matériel nécessaire. Sous le titre "OLIVETTI LETTERA», il fait la remarque suivante : «je ne peux m'en empêcher, j'ai acheté une petite machine à écrire, sans intention littéraire [...]. Cette obsession de taper des phrases -» (MO, p. 20). Frisch commence à raconter son week-end, et le résultat de ce projet autobiographique est le récit Montauk lui-même, à travers lequel Frisch ne se contente pas d'annoncer une nouvelle conception de l'autobiographie dans la littérature, il la met également en ouvre.

La confession qui résulte de son introspection, son intérêt pour sa propre histoire peuvent bien être considérées par Frisch comme une orientation nouvelle dans son écriture, elles constituent néanmoins dans l'usage qu'il en fait un retour au topos de l'autobiographie. Frisch réactualise plusieurs motifs rousseauistes, comme la fuite de la ville vers la nature, la promenade et le temps d'arrêt au bord de l'eau. Il ne faut pas néanmoins analyser le récit de Frisch comme une postfiguration parfaitement fidèle de la promenade rousseauiste : le narrateur autobiographe, Max, ne se trouve que sporadiquement en position de promeneur solitaire : bien au contraire, il jouit de la compagnie de Lynn lors de sa promenade sur la plage. Contrairement à l'île Saint-Pierre, Long Island n'est qu'une presqu'île ${ }^{24}$. Et si Rousseau garde ses pensées inscrites sur de banales cartes à jouer afin d'en tirer la somme dans les Rêveries du promeneur solitaire25, l'obsession d'écrire qui anime Frisch le pousse à acheter une machine à écrire de voyage. La pratique du loisir de Frisch a toutefois ses effets et libère sa créativité littéraire. Lors de ses promenades sur la plage de Montauk, l'auteur

24. Aujourd'hui l'île Saint-Pierre est aussi une presqu'île, mais elle ne le fut qu’à partir de la fin du XIX ${ }^{e}$ siècle en raison de l'ampleur des canaux creusés.

25. Les fragments numérotés, à l'origine griffonnés sur 27 cartes à jouer sont édités dans la Pléiade sous le titre Ébauches des Rêveries (OC, I, p. II65-II72), à ce propos voir aussi les remarques des éditeurs B. Gagnebin et $\mathrm{M}$. Raymond (OC, I, p. I860). 
vieillissant peut se soustraire à son environnement quotidien, s'investir dans le présent et enfin oser poser un regard rétrospectif sur sa propre vie. Dans cette rétrospective, le passage du «il » au « je » n'est pas seulement grammatical. C'est peut-être précisément pour cela que Frisch réussit si bien, par la réappropriation des topoï autobiographiques, à se poser en modèle : parce qu'il introduit une rupture avec la poétologie qu'il prônait jusqu'alors.

\section{Randonnée en montagne}

Montons à présent du niveau de l'Atlantique au sommet des montagnes. À la fois évitées parce qu'elles représentaient une terra incognita inaccessible et dangereuse pour l'homme, et vénérées comme un lieu de contemplation et de méditation, les montagnes se trouvent au cœur d'une tension entre crainte et aspiration dans l'histoire de la culture européenne ${ }^{26}$. Il en est ainsi pour l'ascensionniste le plus remarquable des débuts de l'époque moderne, Pétrarque. Pétrarque marque déjà une étape significative dans l'histoire du loisir car il formule à travers son De Vita solitaria, écrit entre 1346 et I356, l'apologie d'une vie retirée dans l'oisiveté, certainement la plus importante du début de l'époque moderne. On trouve dans cette œuvre de longs développements sur une initiation à l'action morale qui met en évidence les avantages d'une vie consacrée à l'otium sur une vie de negotium. Là aussi, Pétrarque donne des exemples de montagnes, lieux de contemplation et de communication avec Dieu : la montagne du Sinaï, sur laquelle les tables de la loi furent remises à Moïse et la montagne du Carmel sur laquelle Élie fit démonstration du pouvoir de Dieu au peuple ${ }^{27}$. Dans une perspective contemporaine, il importe sans doute de compléter cette liste par saint François d'Assise qui, retiré selon la légende dans un monastère sur la colline de La Verne dans les Apennins, y reçut ces stigmates ${ }^{28}$.

C'est précisément dans la peau d'un ascensionniste que se glisse Pétrarque. Dans une lettre datée du 26 avril I336, qu'il envoie au professeur de théologie parisien, François Denis de Borgo San Sepolcro, Pétrarque rend compte de son ascension du mont Ventoux, le plus haut sommet de Provence. Ici aussi, il

26. Voir P. Raymond, Von der Landschaft im Kopf..., ouvr. cité, p. 6o-66.

27. Voir F. Petrarca, De vita solitaria, éd. G. Martellotti, trad. A. Bufano, Turin, Giulio Einaudi, 1977 [1346-I356], p. I38-I43.

28. Voir B. Vinken, "Erfüllung auf dem Berg. Der heilige Franziskus und der La Verna», dans S. Goumegou, B. Heymann, D. Stöferle et C. Wild (dir.), Über Berge. Topographien der Überschreitung, Berlin, Kadmos, 20I2, p. 63-67. Le sujet des Montagnes sacrées est traité par A. Hahn dans "Berge als lieux de mémoire», dans ibid., p. 22-25. 
prend un modèle : l'ascension du mont Hémos par Philippe $V$ de Macédoine décrite par Tite-Live ${ }^{29}$.

Ce qui le différencie toutefois de tous les prétextes invoqués jusque-là pour justifier sa présence dans la montagne, c'est la qualité de l'expérience décrite : l'Ascension du Mont Ventoux ${ }^{30}$ représente la "première expérience moderne du paysage », parce que Pétrarque y discute de l'apparente absence de but de son entreprise ainsi que de "l'expérience qui joue un rôle précurseur d'un regard désintéressé et purement esthétique sur le paysage qui s'offre à lui comme un spectacle $e^{31} »$. C'est pourquoi la lettre de Pétrarque est considérée comme l'un des récits d'ascension parmi les plus influents, qui marque sans doute en effet le début du genre. Ce faisant, Pétrarque a créé un topos qui influence jusqu'aux autobiographies d'alpinistes modernes.

Au matin d'un jour dont la date n'est pas clairement précisée, Pétrarque se met en route, accompagné de son frère et de deux serviteurs, pour escalader le mont Ventoux. Avec beaucoup de peine et de détours, il atteint avec son frère le plateau au sommet de la montagne. Dans la description de ses impressions, Pétrarque n'emploie nulle part le mot otium, mais il dépeint explicitement une certaine perception de l'espace et du temps qui correspond remarquablement bien à l'archétype du «chronotope du loisir ${ }^{32} »$. Ainsi, il se concentre au début exclusivement sur l'espace qui l'entoure et décrit de façon exhaustive la manière dont cela l'affecte : pour Pétrarque qui, «surpris par cet air étrangement léger et par ce spectacle grandiose», reste "comme frappé de stupeur» $(M V, \mathrm{p} .36)$, la vue du sommet renvoie une impression enivrante et nouvelle.

En revanche, la temporalité de cette expérience des sommets ne joue aucun rôle pour commencer; son je narrant semble tout d'abord être entièrement pris dans le présent et libéré des contraintes et des structures temporelles. La temporalité ne fait son apparition que lorsque Pétrarque commence à mettre en place des références qui renvoient aux montagnes présentant une signification historique ou mythologique : "Je regarde derrière moi : les nuages sont sous mes pieds, et je commence à croire à la réalité de l'Athos et de l'Olympe en voyant de mes yeux, sur un mont moins fameux, tout ce que j'ai lu et entendu à son

29. Voir K. Stierle, «Zwei Bergsteiger. Philipp V. von Makedonien und Petrarca», dans ibid., p. 2.

30. Ce texte se réfere à l'édition de F. Pétrarque, L'Ascension du Mont Ventoux, trad. du latin D. Montebello, éd. P. Dubrunquez, Rezé, Séquences, 1990. (Désormais $M V$.)

3I. K. Stierle, "Zwei Bergsteiger...", art. cité, p. 279.

32. Voir G. Figal, «Muße als räumliche Freiheit. Phänomenologie von Raum und Muße (Heidegger und Nietzsche)», dans B. Hasebrink et Th. Klinkert (dir.), Muße. Konzepte, Räume, Figuren. Der Freiburger Sonderforschungsbereich IoIs im Überblick, Fribourg-en-Brisgau, 20I4, p. I2-I3. 
sujet» ( $M V$, p. 36). Pétrarque compare le Ventoux à des montagnes grecques qui ont une signification importante dans la chrétienté orthodoxe ou dans la mythologie antique et prolonge le cadre de la situation présente jusqu'à le faire remonter aux racines de l'histoire culturelle européenne. Une autre référence historique surgit à la vue des Alpes, qui s'élèvent au loin, «immobiles et couronnées de neige». Elles rappellent à Pétrarque Hannibal, parce que la façon dont «le farouche ennemi du nom de Rome» s'est frayé un chemin à travers la montagne est connue de tous $(M V$, p. 36). À l'aide de ces références soigneusement choisies, Pétrarque s'efforce de charger le lieu de son expérience personnelle d'une signification universelle, de lui donner la valeur d'un lieu de mémoire culturel et de remonter très loin dans la tradition. Pétrarque démontre ainsi combien la représentation de la montagne dans des écrits plus anciens a marqué son propre vécu.

En faisant tendre le récit de son expérience immédiate du lieu vers une réflexion historique sur ce topos, Pétrarque fait le premier pas, comme il le constate lui-même, "qui des lieux me conduit aux époques" ( $M V$, p. 37). Il fait même un pas de plus vers la temporalité :

«Aujourd'hui, me disais-je, cela fait dix ans que tu as laissé tes études de jeunesse et abandonné Bologne : Dieu immortel, éternelle Sagesse, combien de changements dans ta vie durant ces dix dernières années! Si nombreux que je préfère ne pas en parler; d'ailleurs je ne suis pas encore arrivé au port pour évoquer les tempêtes que j'ai essuyées. [...]» Telles sont les considérations que je roulais quand je considérais ces dix dernières années. ( $M V$, p. 37-39)

Le regard contemplatif de Pétrarque trouve un nouvel objet comme source d'auto-bio-graphie, sa propre vie : "Le regard sur l'extérieur se tourne aussi vers l'intériorité du spectateur et fait place à la réflexion ${ }^{33}$ ». Ce passage de la contemplation de l'environnement extérieur à la temporalité intérieure ne peut s'accomplir de façon immédiate. La transition soigneusement orchestrée par Pétrarque, à savoir la référence à un lieu de mémoire commun, à la montagne comme topos mnémonique, est nécessaire. La transition autorise Pétrarque à parcourir subjectivement trois étapes : celle du lieu concret, celle du lieu de mémoire topique, celle du souvenir individuel.

Il est par ailleurs intéressant de voir les arguments avec lesquels Pétrarque remet en question la légitimité de sa digression autobiographique et, ce faisant, formule implicitement les prérequis qu' exige, selon lui, le récit autobiographique. Pétrarque le concède : il ne se trouve pas encore au "port». Ce n’est pourtant

33. P. Oster, "L'air immense ouvre et referme mon livre". Valérys "Cimetière marin" und Petrarcas Mont Ventoux", dans S. Goumegou, B. Heymann, D. Stöferle et C. Wild (dir.), Über Berge. Topographien der Überschreitung, Berlin, Kadmos, 20I2, p. 73. 
qu’à cet endroit que l'activité de mémoire serait légitime. Le port, la fin de la vie, est considéré comme un lieu protégé et remarquablement paisible, mais qui ne peut être atteint qu'à un seul prix : avoir surmonté les peines et les dangers rencontrés sur la mer de la vie. En ce sens, et c'est ainsi qu'il faut comprendre Pétrarque, le droit de jeter un regard en arrière sur sa vie doit d'abord être gagné.

Il s'agit d'une idée qui s'est maintenue dans la littérature et la critique littéraire jusque tardivement au $\mathrm{xx}^{\mathrm{e}}$ siècle. Le chercheur en autobiographie Roy Pascal affirme, encore en 1960, que seuls les hommes et les femmes «of outstanding achievement in life» ("dont la vie représenterait un accomplissement remarquable») seraient en mesure d'écrire des autobiographies d'exception ${ }^{34}$. Il n'y aurait en effet qu'eux qui gagneraient, grâce aux peines endurées durant leur vie, un point de vue solide et sûr qui leur permettrait de porter un regard infaillible sur leur propre vie. Dans la distinction pascalienne entre récit autobiographique légitime et illégitime qui est liée au point de vue adopté, on retrouve une métaphore spatiale dont se sert aussi Pétrarque avec l'image d'un port sûr. Les deux auteurs mettent en avant la catégorie du mouvement : alors que la vie qu'il laisse derrière lui est mouvementée et périlleuse, l'autobiographe se trouve désormais arrivé à un point d'ancrage paisible. Il s'est, dans un sens qui correspond parfaitement à la définition du loisir, extrait des contraintes quotidiennes et se trouve tout à lui.

Un dernier fait attire notre attention. Pétrarque se donne la peine d'emporter un livre jusqu'au sommet, les Confessions de saint Augustin, précisément le livre qui est d'une importance primordiale pour l'appréhension de la vita contemplativa et la praxis du récit autobiographique. Au sommet du Ventoux, Pétrarque sort le livre "pour lire ce qui [lui] tombera sur les yeux" ( $M V$, p. 4I). Pétrarque ouvre par hasard le dixième livre des Confessions et jure que «dès que je fixai les yeux, je lus: Et les hommes vont admirer les cimes des monts, les vagues de la mer, le vaste cours des fleuves, le circuit de l'océan et le mouvement des astres et ils soublient eux-mêmes [...]" (MV, p. 4I).

Dans l'œuvre de saint Augustin, Pétrarque tombe exactement sur le passage qui décrit sa situation présente au sommet de la montagne. Naturellement, il faut prendre en compte le fait que ce hasard est un calcul rhétorique mis en scène par Pétrarque lors de la rédaction de sa lettre ${ }^{35}$. Chez saint Augustin, il

34. R. Pascal, Design and Truth in Autobiography, Londres, Routledge \& Kegan Paul, 1960, p. IO.

35. Cela a contribué dans la critique des années soixante à la popularité de la légende répandue par G. Billanovich : Pétrarque n'aurait jamais escaladé le mont Ventoux, son texte ne serait qu'une compilation réalisée à partir des Confessions de saint Augustin et de l'A $\mathrm{b}$ urbe condita de Tite-Live. Pour une présentation et une réfutation de cette thèse, voir K. Stierle, "Zwei Bergsteiger...", art. cité. 
trouve un précédent au topos qui associe nature et contemplation : l'immensité de la montagne, de l'océan et de l'univers incite le spectateur à faire preuve d'une attention accrue et le transporte dans un mode de perception nouveau qui transcende ce que l'on perçoit de façon immédiate. Et cette perception est, du moins en partie, autobiographique. Saint Augustin est considéré comme le précurseur de l'autobiographie précisément parce qu'il tourne son regard contemplatif non seulement en direction de la création divine, mais aussi sur lui-même, qui fait aussi partie de cet ordre divin ${ }^{36}$. Ainsi, Pétrarque en arrive à la conclusion qu'il "n'est rien d'admirable en dehors de l'âme" ( $M V$, p. 42), alors que la nature devant lui, quand bien même ce serait la vue sur les Alpes enneigées, ne possède à l'inverse qu'une signification secondaire.

À cette pensée, Pétrarque fait immédiatement succéder des actes : «[j]e fermai le livre, furieux de l'admiration que j'éprouvais encore pour les choses terrestres [...]. Alors satisfait jusqu'à l'ivresse de la vue de cette montagne, je tournai les yeux de l'âme vers moi-même $[\ldots]$ " $(M V$, p. 42). De nouveau, Pétrarque détourne son regard du monde extérieur pour rentrer en lui-même. On peut alors saisir le sens de son habile construction intertextuelle : Pétrarque présente la situation de contemplation autobiographique qu'il a lui-même vécue comme une imitatio, comme une postfiguration du topos augustinien. En effet, saint Augustin fournit à Pétrarque une garantie et un argument d'autorité qui permettent de légitimer le fait de faire de sa propre personne le centre de l'attention alors qu'il se trouve devant le spectacle de la création divine. C'est ce déplacement de la contemplation accompli par Pétrarque dans sa lettre sur L'Ascension du Mont Ventoux qui, malgré une structure de base hagiographique, préfigure déjà l'introspection autobiographique. La légitimation de cette entreprise est donnée à Pétrarque par les trois différentes étapes de sa contemplation d'une part (observation du paysage, observation du paysage en tant que lieu de mémoire, considération de ses propres souvenirs), par les Confessions de saint Augustin qui constituent le modèle de l'introspection pétrarquienne d'autre part.

Quelle influence exerce encore à présent le topos du sommet de montagne, lieu de rencontre du monde et du moi dans la littérature contemporaine? L'ascension des montagnes a considérablement gagné en popularité depuis l'âge d'or du XIX ${ }^{\mathrm{e}}$ siècle ${ }^{37}$, comme le montre depuis longtemps déjà le succès des autobiographies d'alpinistes et d'autres livres sur le même thème. Bien

36. Voir M. Wagner-Egelhaaf, Autobiographie, Stuttgart, Metzler, 2005, p. II6-II7.

37. Voir P. Grupp, Faszination Berg. Die Geschichte des Alpinismus, Cologne, Böhlau, 2008, p. 58-66. Pour un regard critique sur l'exaltation de l'alpinisme au xix ${ }^{\mathrm{e}}$ siècle, voir M. Scharfe, Berg-Sucht. Eine Kulturgeschichte des frühen Alpinismus 1750-1850, Vienne, Böhlau, 2007, p. 22. 
sûr, l'ascension des montagnes a pris une toute nouvelle dimension depuis Pétrarque avec l'exploration, jusqu'aux versants nord les plus difficiles puis la conquête des 8 ooo mètres. On peut pourtant montrer qu'il existe une très forte continuité entre les champs sémantiques et les descriptions de l'humaniste de la pré-Renaissance et ceux d'un alpiniste pop-star d'aujourd'hui tel que Reinhold Messner.

Messner se prête à cette comparaison non seulement à cause de ses nombreux et extraordinaires exploits en montagne et le renouvellement de l'alpinisme de l'extrême dont il est à l'origine depuis les années soixante-dix, mais aussi en raison de l'énorme succès de ses publications. Messner répand à travers ses livres une philosophie de l'alpinisme, qui recourt largement aux topoï du loisir, ce qui contribue probablement à son succès éditorial :

Alors je vais en avant. Sans pour autant poursuivre un but particulier. Et j'adore ça, aller seul, être seul avec moi-même. Je n'apprécie pas quand quelqu'un marche devant ou derrière moi. En chemin, j'aime bien parler avec moi-même, je trouve mon chemin à l'instinct et alors je peux arrêter de penser. Avec cela, la possibilité de marcher les bras écartés à travers ce monde hostile. Ce calme ${ }^{38}$ !

Pour Messner, l'alpinisme se caractérise par l'absence de but imposé, la marche solitaire et le monologue, un programme digne de Rousseau ${ }^{39}$. L'alpinisme serait par conséquent libéré de toute attente utilitariste : «Non, cela n’apporte rien à l'humanité : gravir le mont Everest sans bouteille d'oxygène et aller seul sur le Nangat Parbat. Mais qui dit que nos actions doivent rapporter quelque chose?» (WE, p. II4) Rousseau conférait à ses promenades sur l'île Saint-Pierre le statut de praxis idéale, dans laquelle le sujet humain ne se réjouit de rien d'autre que de lui-même, où l'"on se suffit à soi-même comme Dieu» (OC, $I$, p. I047). Ce topos de la constitution et de la réalisation du sujet dans le loisir est également transposé dans l'alpinisme par Messner. Il obéit uniquement au "désir d'atteindre le but qu'il s'est lui-même fixé» (WE, p. IIs) et ne veut qu' «être accepté comme celui [qu'il est]» (WE, p. II5). L'alpinisme lui permet en outre d'atteindre quelque chose "qui fait à tous défaut : être en accord avec soi-même» (WE, p. II6).

38. R. Messner, Die weisse Einsamkeit. Mein langer Weg zum Nanga Parbat, Munich, Malik, 2003, p. 127. (Désormais WE.)

39. Messner n'est naturellement pas le premier à mettre en avant l'absence de finalité extérieure dans l'alpinisme. Bien au contraire, la finalité intrinsèque à l'activité fut décrite autour de 1900 comme une caractéristique essentielle de l'alpinisme par les premiers historiens qui se sont penchés sur ce sujet et même considérée comme la césure entre l'alpinisme moderne, qui à partir des environs de I840/1850 trouvait précisément son but en lui-même, et le "préalpinisme» qui l'a précédé. (Scharfe, Berg-Sucht..., ouvr. cité, p. 22.) 
Aristote déjà décrivait la scholè comme une situation dans laquelle on se suffit à soi-même; Rousseau et ses contemporains remettent cette pensée au goût de l'époque moderne en la plaçant sous le signe de l'esthétique moderne de l'autonomie : l'artiste et l'écrivain trouvent dans le loisir un espace libre pour créer leur œuvre de façon entièrement autonome, sans être tributaires d'intérêts extérieurs. Ici aussi, l'exposé de Messner s'inscrit dans la droite lignée de Rousseau, lorsqu'il explique : "Je ne refuse pas seulement les moyens techniques en montagne, comme on refuserait une béquille, j'aspire à un très haut degré d'autonomie. Pour moi, il s'agit d'indépendance. Au moins pour une courte durée, là-haut, se suffire à soi-même et tout faire soi-même.»(WE, p. I42)

Enfin, Messner reconnaît dans l'alpinisme une activité qui se distingue par une "certaine incertitude», la même qui caractérise les situations de loisir ${ }^{40}$ : "Je sais ce qui m’attend et pourtant, tout reste possible. Réduire au minimum l'imprévisible et malgré tout vivre avec le mystère.» (WE, p. I42) Ainsi, Messner dépeint l'alpinisme comme un art du possible : en d'autres termes, comme une praxis du loisir. Même s'il n'emploie pas le concept de loisir, les points communs et les analogies systématiques avec d'autres descriptions du loisir justifient une telle affiliation.

Les passages de l'ouvrage cités ici sont tirés de l'ouvrage de Messner, Die weisse Einsamkeit. Mein langer Weg zum Nanga Parbat (La Solitude blanche. Ma longue route vers Nanga Parbat), qui se présente en partie comme un livre documentaire sur l'histoire de l'ascension du Nanga Parbat et en partie comme une autobiographie. À côté de développements généraux sur l'histoire de l'alpinisme, Messner y décrit également l'expérience qu'il a lui-même faite sur ce sommet de 8 I25 mètres qui ne représente pas seulement son premier 8 ooo mètres, mais auquel le lie sa destinée personnelle. En 1970, Reinhold Messner fait pour la première fois l'ascension du Nanga Parbat en compagnie de son frère. On trouve ici un parallèle anecdotique avec Pétrarque, qui est également parti en compagnie de son frère. Mais à l'inverse de l'excursion sur le mont Ventoux, peu dangereux en comparaison, les événements sur le Nanga Parbat s'enchaînent de façon si malheureuse que Günter Messner, le frère de Reinhold, perd la vie lors de la descente. Ses concurrents et tout particulièrement le guide de l'expédition Karl Herrligkoffer rendent Reinhold Messner responsable de cette erreur mortelle. En réaction à cette accusation, celui-ci ne cessera, dans ses divers ouvrages, de raconter la façon dont il a vécu l'expédition. Dans ses efforts pour se justifier et pour exorciser son traumatisme,

40. Voir H.-G. Soeffner, "Muße - Absichtsvolle Absichtslosigkeit», dans B. Hasebrink et P. Ph. Riedl (dir.), Muße im kulturellen Wandel. Semantisierungen, Ähnlichkeiten, Umbesetzungen, Berlin-Boston, De Gruyter, 20I4, p. 46. 
Messner confère à l'ascension du Nanga Parbat le statut de narration la plus importante de sa vie.

Sur cet arrière-plan, Die weisse Einsamkeit raconte la seconde ascension du Nanga par Messner en 1978 après plusieurs tentatives manquées. (Cette victoire sur le sommet est entrée dans les livres d'histoire comme la première ascension en solo complète d'un sommet de 8 ooo mètres.) Messner, après s'être battu seul durant plusieurs jours à travers «l'immensité blanche», décrit ce qui s'est passé au sommet de la façon suivante :

Il est $16 \mathrm{~h}$ 30. Je sors un étui en aluminium de mon anorak, plante un piton dans la première fissure que je peux atteindre de là où je suis assis et $y$ attache l'étui avec une ficelle. Puis j'extirpe un parchemin de l'une de mes poches, y griffonne la route, la date et mon nom. C'est comme un rituel. Comme je l'ai aussi fait des milliers de fois sur les sommets des Alpes. (WE, p. 174)

Sur le parchemin que Messner abandonne sur le sommet dans un étui d'aluminium, se trouve une reproduction de la première page d'une bible de Gutenberg, comme le montre une illustration du livre. À l'instar de Pétrarque, Messner emmène un texte religieux avec lui sur le sommet : la Genèse. Mais au lieu d'en lire un passage, il inscrit les dates de son expédition sur le parchemin. Il s'agit expressément d'une activité habituelle et ritualisée, ce qui ne change toutefois rien à l'audace de cette mise en scène de soi : Messner désacralise la Genèse pour en faire un palimpseste en superposant à l'acte de création divin son propre exploit. Mais il faut aussi comprendre cette superposition comme un ancrage dans une tradition textuelle: Messner n'est pas le seul à se confronter à la puissance de la Création; comme chez Pétrarque, la tradition culturelle et la préfiguration d'une rencontre entre le moi et le monde sous une forme textuelle accompagnent l'auteur au sommet de la montagne.

Bien qu'il ne lui reste que peu de temps, puisqu'il doit être rentré au camp avant la tombée de la nuit, Messner décrit ainsi les minutes passées au sommet comme un temps d'arrêt : «Le monde est silencieux.» (WE, p. I74) Même l'impératif de temps qui pourrait lui être fatal perd, momentanément du moins, sa signification. Exception faite des hauteurs qui se trouvent au-delà des 8000 mètres et dont l'air est pauvre en oxygène, l'alpiniste trouve dans la montagne un refuge paisible et sûr, et même un point d'ancrage immobile et intemporel, qui, en accord avec la thématique de l'autobiographie, constitue l'une des conditions nécessaires à l'introspection : «Je ne peux pas m’arracher aux sommets. [...] après avoir quitté une mer de solitude pour monter dans le refuge que m'offre l'univers, un sentiment de plénitude m'habite une fois arrivé au sommet» (WE, p. 176-I77).

Concrètement, ce temps d'arrêt s'ouvre sur un sentiment d'adéquation à soi-même, qui correspond à la perfection aux critères stéréotypés de l'autobiographie classique : "Mes idées et moi ne font qu'un.»(WE, p. 174) La 
«force des sentiments» éprouvés au sommet ne peut pourtant être exprimée en fin de compte : «tout cela est au-delà des mots». Messner ne trouve qu'un seul mot pour briser son silence : " $I c h$ ", dis-je; mais rien que ce simple son, échappé de ma gorge, menace de me déchirer» (WE, p. I77). Messner fait de la rencontre avec soi-même le but véritable de son expédition en haute montagne. Tout comme chez Pétrarque, le changement de perspective s'accomplit par le passage du monde extérieur à une temporalité personnelle, c'est-à-dire à une réflexion autobiographique. Cela n'est possible que sur le sommet qui a coûté la vie à son frère : "Mon être, mon histoire personnelle se déploient devant moi au gré du vent.» (WE, p. I77)

La représentation a posteriori que Messner fait de son expérience des sommets, souvent chargée de pathos, n’a rien à envier à celle de Pétrarque. La façon dont il stylise et met en scène son récit selon un modèle stéréotypé est très nettement identifiable. Peu importe de savoir si Messner a lui-même lu Pétrarque. La continuité dans la stylisation littéraire prouve l'existence des processus de réception culturelle et de postfiguration qui la sous-tendent. Ainsi Pétrarque et Messner ont, malgré les six siècles qui les séparent, principalement recours au même topos : c'est précisément dans l'isolement extrême offert par la nature que survient un temps d'arrêt, un moment de loisir, dans lequel réflexion autobiographique et autoconstitution du sujet ont des chances de succès. 